\title{
Evolutionary topology optimization extended finite element method and isolines
}

\author{
Meisam Abdi, Ricky Wildman and Ian Ashcroft
}

Faculty of Engineering, University of Nottingham, University Park, Nottingham, UK

eaxma5@nottingham.ac.uk,Ricky.Wildman@nottingham.ac.uk,Ian.Ashcroft@nottingham.ac.uk

\begin{abstract}
This study presents a new algorithm for structural topological optimization of 2D continuum structures by combining the Extended Finite Element Method (X-FEM) with an evolutionary optimization algorithm. Taking advantage of an isoline design approach for boundary representation in a fixed grid domain, X-FEM can be implemented to improve the accuracy of FE solutions on the boundary during the optimization process. Although this approach doesn't use any remeshing or moving mesh algorithms, final topologies have smooth and clearly defined boundaries which need no further interpretation. Numerical comparisons of the converged solutions with standard BESO solutions show the efficiency of the proposed method and comparison with the converged solutions using MSC NASTRAN confirms the high accuracy of this method.
\end{abstract}

Keywords: Topology Optimization, X-FEM, ESO, Fixed grid, evolutionary

\section{Introduction}

In recent years, structural optimization has become a rapidly growing field of research, with application in many areas such as mechanical, civil and automotive engineering. Topology optimization is one of the most challenging aspects of structural optimization, in which one needs to find the best topology as well as shape of a design domain. The approaches that have been proposed for the topology optimization of continuous structures fall into two categories: first, mathematical based methods such as homogenization (Bendsøe and Kikuchi, 1988), Solid Isotropic Material with Penalization (SIMP) (Bendsøe, 1989; Zhou and Rozvany, 1991) and level set method (Wang et al., 2003; Allaire et al, 2004); second, heuristic methods which are more intuitive and less 
mathematical, such as evolutionary structural optimization (ESO/BESO) methods (Xie and Steven 1993; Querin et al, 1998; Yang et al, 1999).

ESO is based on the assumption that the optimal layout of the design domain can be obtained by gradually removing inefficient material from the design domain (Huang and Xie, 2009). In the original ESO method, the elements of the design space were ranked in terms of their sensitivity, and those with lower sensitivity were removed from the design domain until a converged solution was obtained. Bi-directional evolutionary structural optimization (BESO) is an extension of ESO in which the elements are allowed to be added and removed simultaneously. These heuristic methods are easy to program and provide a clear topology (no grey regions of intermediate densities as in SIMP) in the resulting optimal designs. Conventional ESO/BESO algorithms have been successful since they can be easily combined with the finite element model of a structure. However they suffer from a weak capability of boundary representation as they are defined by the finite element mesh, which is non-optimal with respect to the final converged solution. This limitation causes difficulties in combining these methods with CAD and the obtained solutions require post processing to manufacture a design with smooth and manufacturable surface.

The fixed grid finite element method (FG-FEM) allows the boundaries of the design to cross over finite elements. This capability has been used in boundary based optimization methods such as the level set method, and element based optimization methods, such as fixed grid evolutionary structural optimization (FG-ESO). FGESO or Isoline/Isosurface approach (Victoria et al, 2009; Victoria et al, 2010) is an alternative to ESO in which the inefficient material is allowed to be removed/added within the elements of the design domain during an evolutionary process. The boundaries are defined by the intersection of the isoline plane with the criteria distribution of the design domain. Since in this approach the boundary of the design is no longer consistent with the fixed finite elements as in ESO, a classical finite element analysis may result in a poor FE approximation on the boundary. Conventionally in the fixed grid finite element approach, the element stiffness is assumed to be proportional to the area fraction of the solid material within the element (also called the density scheme). Although this approach is widely accepted and implemented in many works (Allaire et al, 2004; Victoria et al, 2009), studies have shown that it cannot provide accurate results for the boundary elements (Dunning et al, 2008; Wei et al, 2010).The Extended finite element method (X-FEM) is another fixed grid approach which can be used to model void/solid interfaces. X-FEM extends the classical finite element approach by adding special shape functions which can represent a discontinuity inside finite elements. In this approach, the geometry of the discontinuity is often described by a level set method. A combination of the level set description of the geometry 
and the fixed mesh framework of X-FEM has been used in recent level set based topology optimization work (Wei et al, 2010; Miegroet et al, 2007).

This study presents a simple and effective evolutionary optimization approach in a fixed grid domain. The novelty of this work is to apply X-FEM to the evolutionary optimisation algorithm. The proposed method doesn't require a level set framework for geometry description in the X-FEM and the boundaries of the design can be simply represented by isolines of a desired structural performance. The algorithm is implemented in the topology optimization of two test cases and the final solutions are compared to standard BESO solutions. To evaluate the accuracy of the proposed method, the solutions are imported to NASTRAN and reanalysed using the classical finite element method.

\section{Fixed grid approach}

The fixed-grid method is a technique to model the boundaries with a non-conforming mesh. It has been used for solving the problems with moving boundaries such as those requiring structural optimization problems. Unlike the remeshing methods in which the design domain, or a narrow area around the boundary, is remeshed in every iteration, the fixed grid FEM doesn't require the time consuming remeshing process and can be easily implemented. In this method the real structure is superimposed on the fixed finite elements of the design space and makes three types of elements: the solid elements which lie inside the structure (S elements), the void elements which lie outside the structure (V elements) and the elements which lie on the boundary of the structure (B elements), as shown in figure 1.

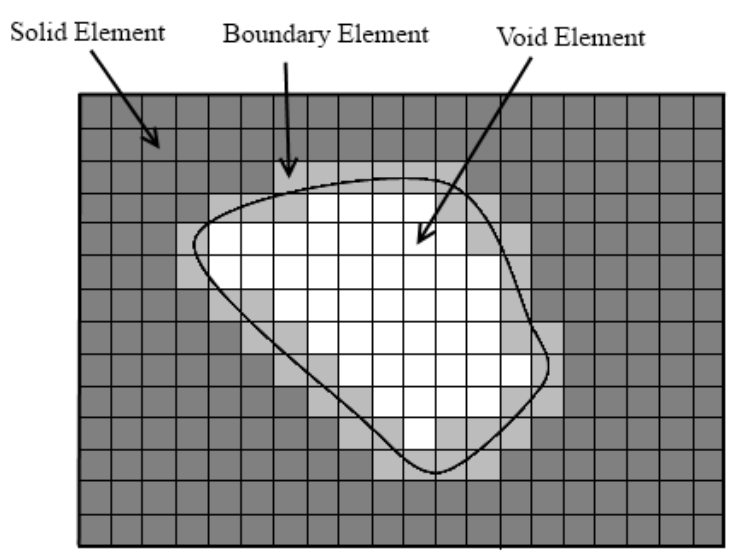

Figure 1. A material/void interface problem in a fixed grid. 


\subsection{Density scheme}

In a conventional fixed grid approach, the stiffness matrix of boundary elements is approximated by a density scheme in which the stiffness of the element is proportional to the area ratio of the solid part of the element:

$\xi^{(e)}=\frac{A_{s}^{(e)}}{A_{s}^{(e)}+A_{v}^{(e)}}=\frac{A_{s}^{(e)}}{A_{t}^{(e)}}$

$K_{B}=K_{S} \xi^{(e)}$

where $A_{s}^{(e)}, A_{v}^{(e)}$ and $A_{t}^{(e)}$ represent the solid area, void area and the total area of the element, respectively, and $K_{B}$ and $K_{S}$ are the stiffness matrices of the boundary element and solid element, respectively.

In the density scheme, the material is considered to be uniformly distributed through the whole element and the variations in material distribution in an element are not taken into account in calculating the element stiffness matrix. For example, figure 2 shows three different shapes for a boundary element where the area fraction of solid material within the element is 0.50 . Using the density method (equation 2) the same stiffness is calculated for all three elements. This method may cause errors near the boundary of the design during the optimization process.
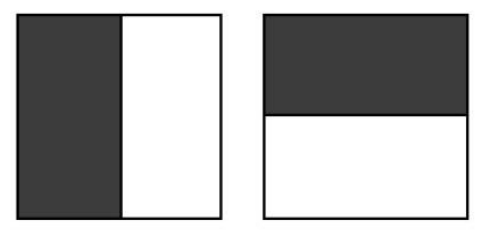

a

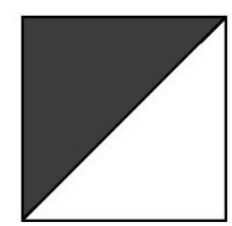

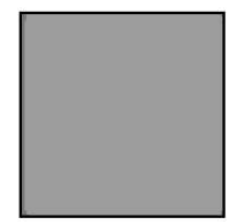

b

Figure 2. (a) Typical boundary elements for area ratio $=0.50$. (b) Their density scheme equivalent solid element with $50 \%$ density.

\section{2. eXtended Finite Element Method}

The extended finite element method (X-FEM) (Belytschko and Black, 1999; Moës et al, 1999) is an alternative fixed grid approach in which the classical finite element approximation is enriched by special functions through the concept of partition of unity (Melenk and Babuška, 1996). X-FEM was originally developed to represent crack growth in a fixed grid domain. Using traditional FEM for the simulation of crack propagation is very challenging because of the continuous changes in the topology of the domain. The application of X-FEM for this case has been very successful because the FE mesh can be generated independent of the geometry of the crack, and remeshing is not required during crack propagation. For crack modelling, the idea of X-FEM is to use additional degrees of freedom in the usual FE spaces by adding a discontinuous function (Heaviside step function) and the asymptotic crack tip displacement fields to the conventional FE displacement field. Therefore 
the standard finite element approximation is extended by adding enrichment functions to the continuous displacement field and defining additional degrees of freedom for the nodes which support the discontinuity:

$u(x)=\sum_{i} u_{i} N_{i}(x)+\sum_{j} a_{j} N_{j}(x) H(x)$

In the above equation, the function on the right hand side shows the conventional finite element approximation of the displacement field in an element where $N_{i}$ are the classical shape functions associated to the nodal degrees of freedom, $u_{i} . N_{j}(x) H(x)$ supported by enriched degrees of freedom, $a_{j}$, are discontinuous shape functions constructed by multiplying a classical $N_{j}(x)$ shape function with a Heaviside function $H(x)$ presenting a switch value where the discontinuity lies. X-FEM has also been implemented for other kinds of discontinuities such as fluid/structure interaction (Gerstenberger and Wall 2008) and modelling holes and inclusions (Sukumar et al 2001). In our case the X-FEM scheme for modelling holes and inclusions can be implemented for modelling material/void interfaces during the optimization process. In this approach, the displacement field is approximated by the following equation

$u(x)=\sum_{i} N_{i}(x) H(x) u_{i}$

where the Heaviside function $H(x)$ has the following properties

$H(x)=\left\{\begin{array}{ll}1 & \text { if } x \in \Omega \\ 0 & \text { if } x \notin \Omega\end{array}\right.$.

which implies that the value of Heaviside function is 1 for the nodes outside the void and 0 for the nodes in the interior of the void. Since there is no enrichment in the displacement approximation equation of X-FEM in modelling holes and inclusions, there will be no augmented degrees of freedom during optimization. The proposed X-FEM integration scheme will be discussed in next sections.

\section{Structural optimization problem}

The topology optimisation problem where the objective is to minimize the strain energy can be written as

Minimize: $c=\frac{1}{2} U^{T} K U$

Subject to: $\frac{\sum_{e=1}^{N} v_{S}^{(e)}}{V_{0}}=V^{*}$

where $c$ is the total strain energy, and $U$ and $K$ are the global displacement and global stiffness matrices, respectively. $N$ denotes the number of finite elements in the design domain. $v_{S}^{(e)}$ is the volume of the solid part 
of the element, $V_{0}$ is the design domain volume and $V^{*}$ is the prescribed volume fraction. While in ESO/BESO methods, the presence/absence of each element in the design domain is considered as a design variable, in our proposed method the material distribution inside each element is considered as a design variable. Strain energy density (SED) is a reliable criterion to indicate inefficient use of material in a design space (Huang and Xie, 2009). Elemental SED can be obtained from

$S E D_{e}=\frac{1}{2} u_{e}^{T} k_{e} u_{e} / v_{e}$

with $u_{e}$ the element displacement vector and $k_{e}$ the element stiffness matrix, which is calculated using an XFEM scheme. The topology optimization operates by gradually removing the material from low SED regions and adding it to high SED regions during an evolutionary procedure. The effective removal/redistribution of material within the design domain can be achieved through an isoline topology optimization approach by assigning a weak material property to low SED regions and solid material property to high SED regions (softkill scheme).

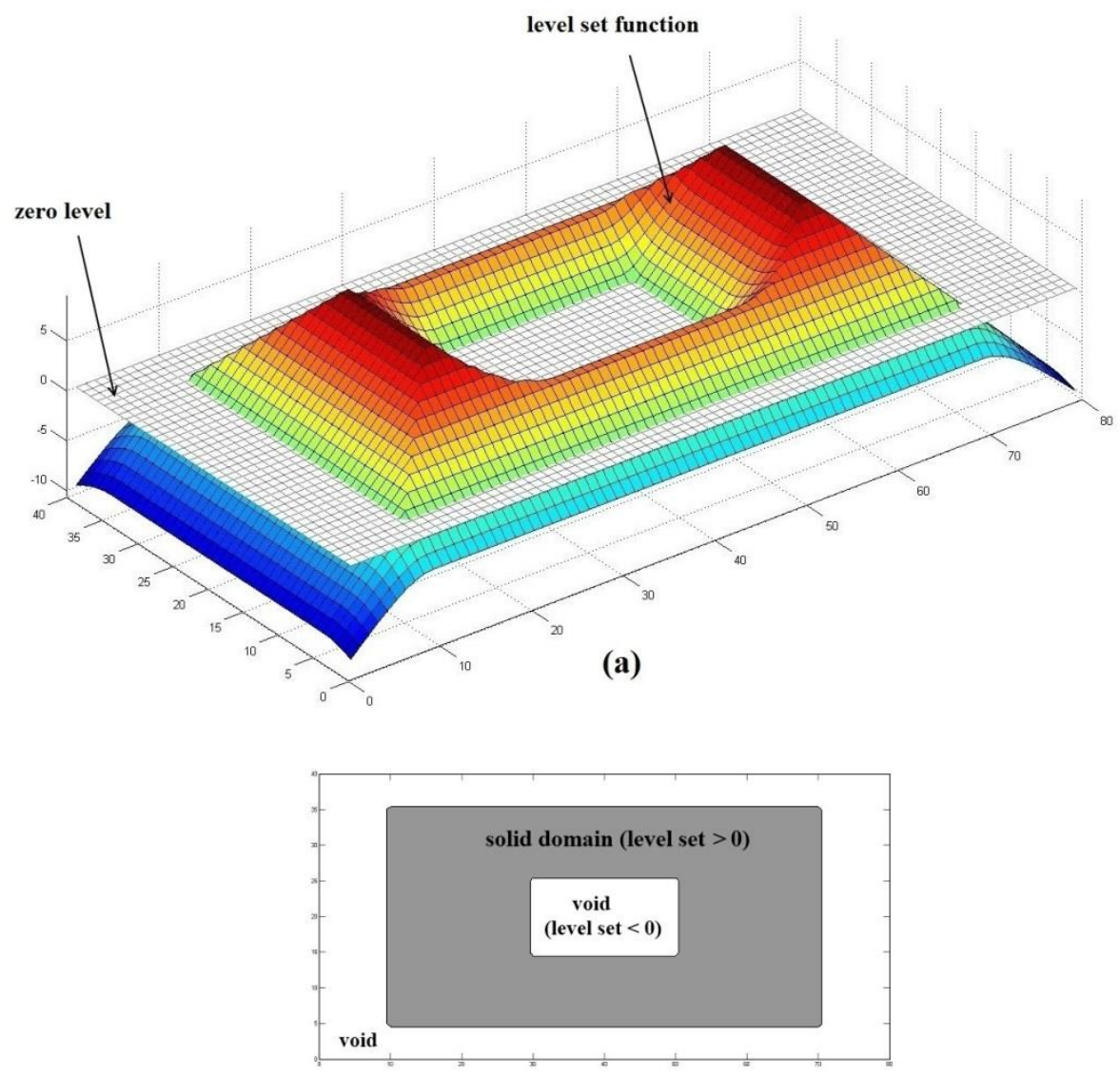

(b)

Figure 3. Level set description of a 2D design. (a) 3D level set function (signed distance function) (b) 2D design domain with solid and void phases. 


\subsection{Isoline topology optimization}

The basic idea of isoline design is to represent the shape and topology of the structure using the contours of the desired structural behaviour. This idea has been suggested in several studies (Maute and Ramm, 1995; Lee et al, 2007). The isoline boundary representation differs from the level set description of the design boundary. In the level set method the boundary is described by a zero level set function (usually signed distance function) (figure 3) and the evolution of boundary is represented by solving so called 'Hamilton-Jacobi' equation. In the isoline method, the design boundary is represented by a minimum level of a criterion (such as von Mises stress or SED) which is iteratively updated during the design process (figure 4). The isoline optimization algorithm that is used in this paper is originated from isoline topology design (ITD) algorithm proposed by Victoria et al (2009). Unlike ESO/BESO methods in which the optimization operates at an elemental level, in the ITD approach, the optimization is performed in a global level, based on structural performance. In our study, the distribution of the desired structural behaviour is obtained in the analysis phase through the use of X-FEM.

The ITD approach can be summarized into the following steps:

1- An extended finite element analysis is performed to find the distribution of strain energy density within the design domain.

2- A minimum SED level (MSL) is determined and the new structural boundary is obtained from intersection of SED distribution and MSL.

3- The regions of the domain having the criteria (SED) level less than MSL are not included in the design domain. Therefore their material property is set to the weak material. The regions where the criteria level are greater than MSL are inside the design domain and their material property is set to the solid material.

Steps 1-3 are repeated by gradually increasing the MSL until a desired optimum is obtained. 

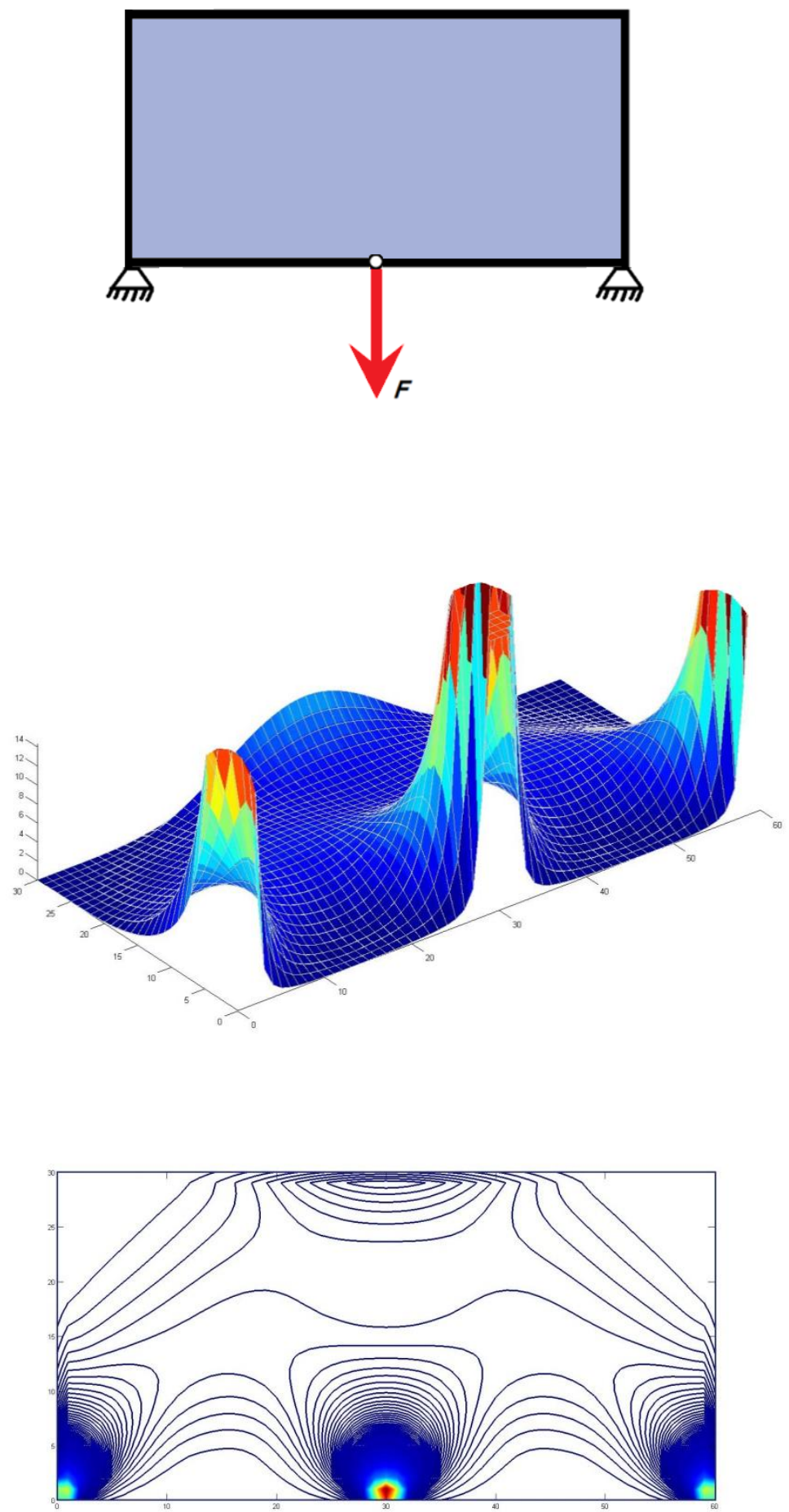

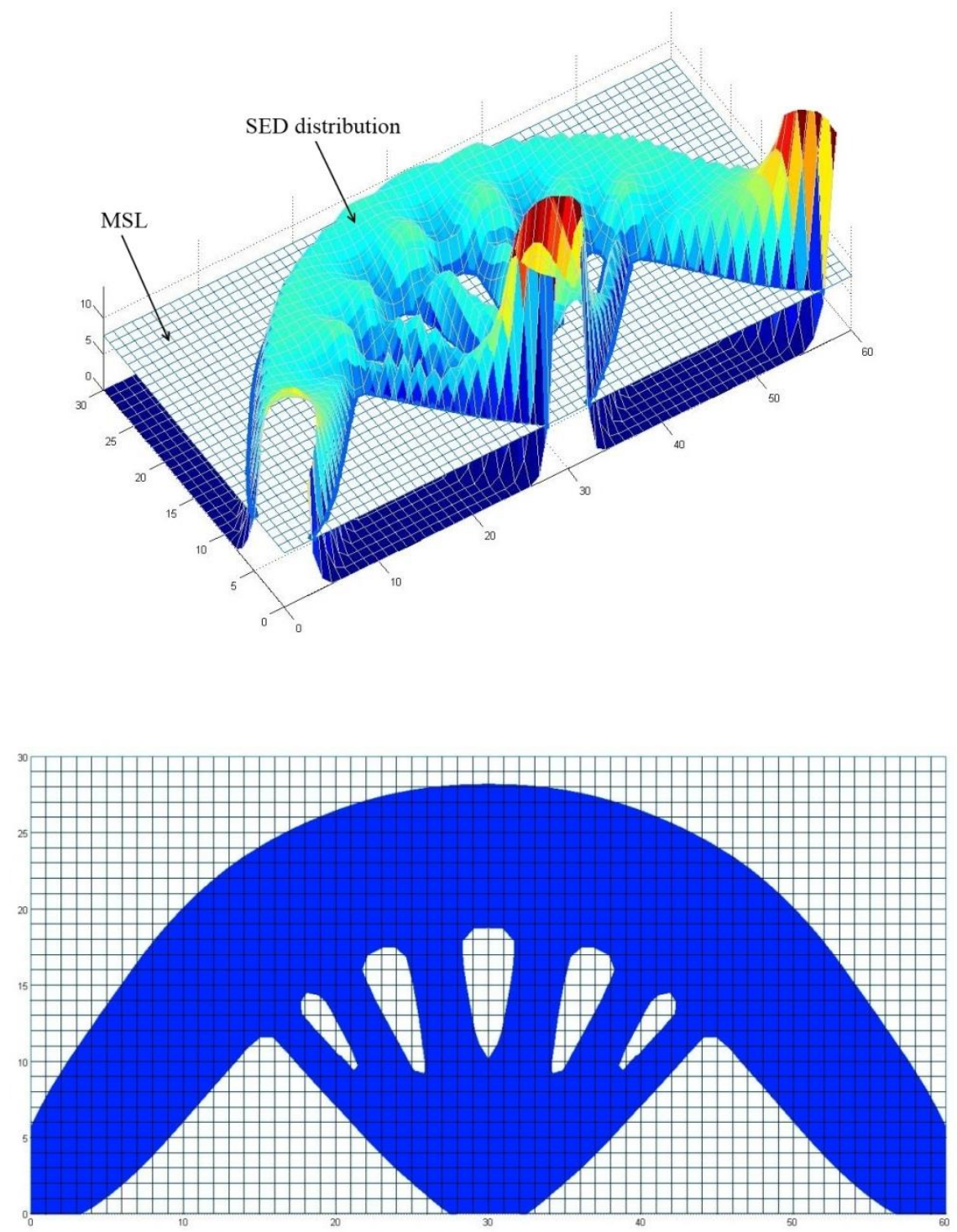

Figure 4. (a) Initial design domain with boundary conditions. (b) SED distribution on the initial design domain. (c) Isolines of strain energy density (initial design domain). (d) Structural boundary represented by intersection of criteria (SED) distribution and MSL at volume fraction $=0.5$. (e) Final solution shown in a fixed grid domain (volume fraction $=0.5$ ).

\subsubsection{The evolutionary procedure in isoline topology optimization}

The nodal level of SED in any part of the design domain can be obtained by performing an extended FE analysis and comparing the SED of that node with the maximum SED of the entire domain. Using the nodal SED level, the elements are categorized into three groups: void elements, solid elements and boundary elements:

void element if, for $j=1: 4, \quad \frac{S E D_{j}}{S E D_{\max }}<R F_{i}$

solid element if , for $j=1: 4, \quad \frac{S E D_{j}}{S E D_{\max }}>R F_{i}$ 
where $R F_{i}$ is the current redistribution factor $(R F)$, and $j$ denotes the element's node number. The boundary of design can be obtained by the intersection of the SED distribution and the minimum SED level, which is calculated by

$M S L=R F_{i} \times S E D_{\max }$

With the current redistribution factor, the iterative process of the extended finite element analysis and material removal/redistribution takes place until the change in volume fraction is less than a minimum value $\Delta V$, which means that a steady state is almost reached. An evolutionary rate, $E R$, is added to the redistribution factor, such that

$R F_{i+1}=R F_{i}+E R$.

With the new redistribution factor, the extended finite element analysis and material removal/redistribution is repeated until a new steady state is reached. The evolutionary process continues until a desired optimum, such as a final volume fraction or a maximum strain energy level, is reached. In this study, the volume fraction and the objective function (total strain energy) are used to check the convergence. The evolutionary process continues until the volume fraction condition is satisfied. From this time, the optimization process runs with a constant redistribution factor (zero evolution rate) until the changes in the objective function in the last 5 iterations are within a $0.1 \%$ tolerance.

The number of iterations in the evolutionary process in the proposed method is affected by the value of evolution rate as well as the maximum strain energy density $\left(\mathrm{SED}_{\max }\right)$. Selecting a high evolution rate can reduce the computational time. However, very high values of evolution rate may result in local optima or nonconvergent solutions. A typical value for the evolution rate can be obtained from

er $=0.01 \times \frac{S E D_{\text {ave }}}{S E D_{\max }}$

where $S E D_{\text {ave }}$ is the average SED for the fully solid design domain.

\subsection{X-FEM integration scheme}

Equation 4 defines a zero displacement field for the void part of the element, which means that only the solid part of the element contributes to the element stiffness matrix. Thus we can use the same displacement function as FEM (first term in equation 3) and simply remove the integral in the void sub-domain of the element:

$K_{e}=\int_{\Omega_{S}} B^{T} D_{S} B t d \Omega$ 
where $\Omega_{S}$ is the solid sub-domain, $B$ is the displacement differentiation matrix, $D_{S}$ is the elasticity matrix for the solid material and $t$ is the thickness of the element. When an element is cut by the boundary, the remaining solid sub-domain is no longer the reference rectangular element. In order to numerically calculate the integral given by equation 13 , the solid part of the boundary element is partitioned into several sub-triangles (figure 5) and the Gauss quadrature method is used:

$K_{e}=\sum_{i=1}^{n} \int_{T_{i}} B^{T} D_{S} B t d \Omega$

where $n$ is the number of sub-triangles inside the element and $T$ denotes the triangle domain.

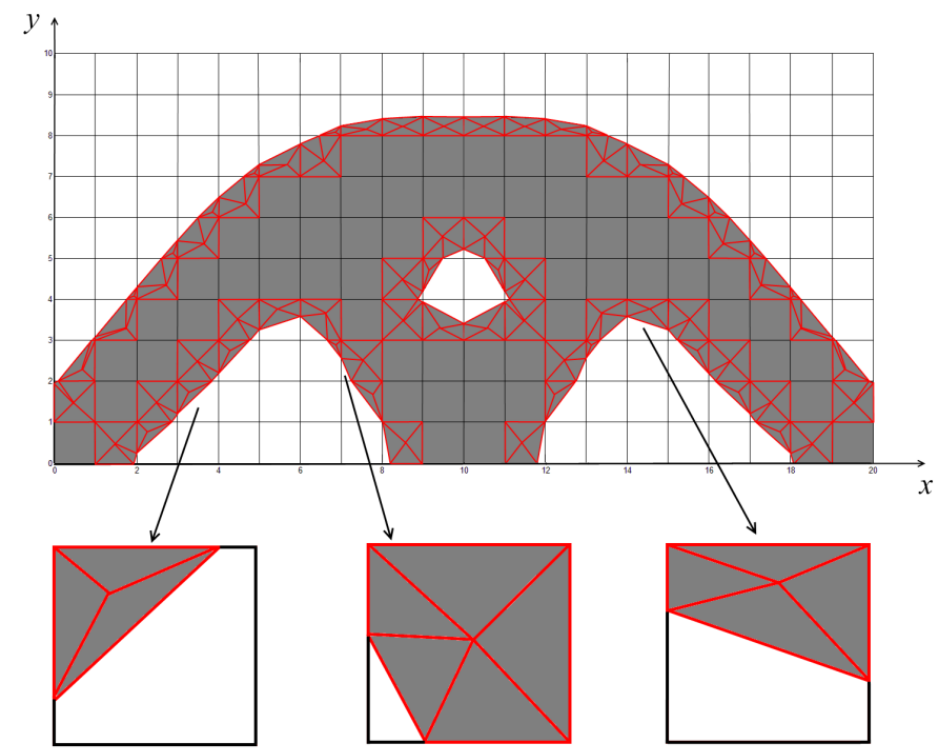

Figure 5. The solid sub-domain of the boundary elements are partitioned into several sub-triangles.

\subsubsection{Triangulation of boundary elements}

The topology and shape of a boundary element can be found using the values of nodal relative SED which is defined by:

$S E D_{\text {rel }}^{j}=S E D^{j}-M S L$

where $j$ denotes the node number. Nodes having negative relative SED belong to the void part of the domain and nodes with positive relative SED are located in the solid domain. Therefore, an element which has at least one node with negative $\mathrm{SED}_{\text {rel }}$ and one with positive $\mathrm{SED}_{\text {rel }}$ is a boundary element. 
The intersection point of the boundary and element edge between two neighbouring nodes $i$ and $j$ can be found using bilinear interpolation of nodal $\mathrm{SED}_{\text {rel }}$ and shape functions:

$x_{i}=\frac{l_{i j}}{1-\frac{S E D_{r e l}^{i}}{S E D_{r e l}^{j}}}$

where $x_{i}$ is the distance between node $i$ and the intersection point, and $l_{i j}$ is the element length between the nodes $i$ and $j$. Depending on its topology, a boundary element may have 2, 3 or 4 intersection points. The sub-triangles can be defined by defining an extra point inside the solid subdomain of the element (typically in the centre of solid area) and connecting it to the solid nodes as well as the intersection points. Figure 6 shows a boundary element with two intersection points and typical values for $\mathrm{SED}_{\text {rel }}$.

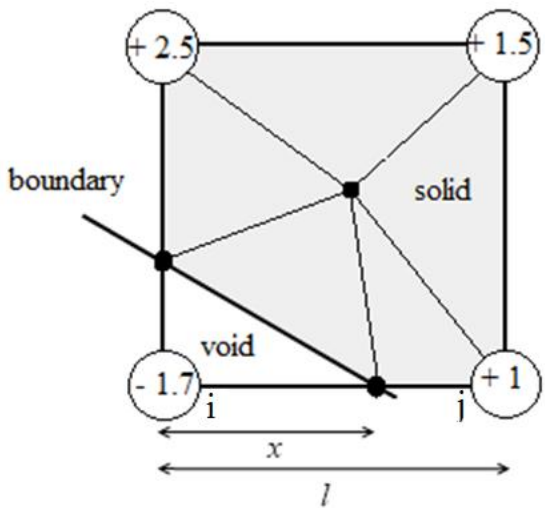

Figure 6. A boundary element with typical values for nodal relative SED

\subsubsection{Gauss quadrature method}

In order to apply the Gauss quadrature method to triangles, the two dimensional integrals in terms of the physical coordinates are transferred to the triangle's natural coordinates and represented as a series of weighted functions:

$\iint_{\Omega_{e}} F(x, y) d x d y=A_{T} \sum_{i=1}^{m} W_{i} F\left(\xi_{1}^{i}, \xi_{2}^{i}, \xi_{3}^{i}\right)$

where $m$ is the number of gauss points, $\xi$ the coordinates of the gauss points, and $A_{T}$ is the area of the triangle. Substituting equation 17 into equation 14, the element stiffness matrix can be obtained by 
$K_{e}=\sum_{i=1}^{n} \sum_{j=1}^{m} A_{T} W_{j} f\left(\xi_{1}^{j}, \xi_{2}^{j}, \xi_{3}^{j}\right)$

where

$f=B^{T} D_{S} B t$

with $n$ the number of sub-triangles in the solid domain of the element. In our study, the second order gauss rule with 3 midline gauss points was implemented (figure 7). To validate this X-FEM scheme, we calculated the stiffness matrix of a fully solid rectangular element having the Young's modulus $E=1$ and the Poisson's ratio $v=0.3$, by 2 different approaches: first, using the classical finite element approximation; second, using the $\mathrm{X}$ FEM scheme described above in which the element is divided into sub-triangles and integration is performed using gauss quadrature for triangles, as shown in figure 8. Both methods resulted in exactly the same stiffness matrix for the element, thus validating the X-FEM scheme.

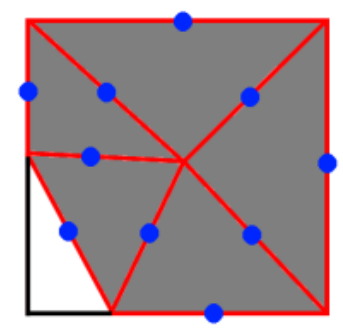

Figure 7. X-FEM integration scheme.

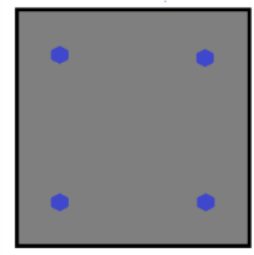

(a)

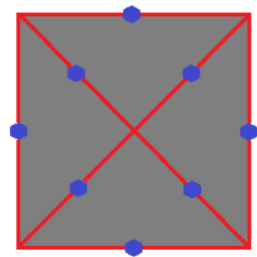

(b)

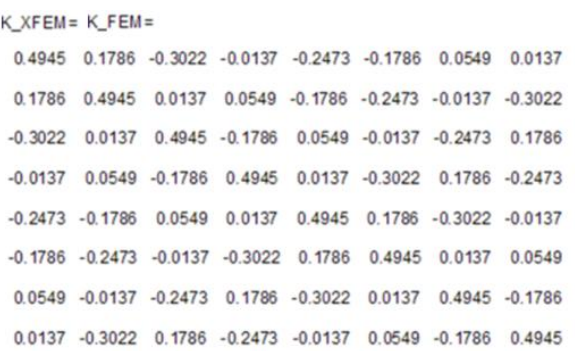

(c)

Figure 8. (a) A solid element represented by classical FEM. (b) The solid element represented by our X-FEM scheme. (c)

The stiffness matrix obtained using both FEM and X-FEM approaches.

\subsection{Combining X-FEM and the optimization algorithm}

Figure 9 illustrates the topology optimization procedure used, which in general consists of the following steps:

1- Initialization: in this step, the dimensions of the design domain, fixed grid mesh and initial material distribution within the design domain are defined; boundary and loading conditions are applied and the 
parameters of the optimization algorithm, such as evolution rate (ER) and final volume fraction, are defined.

2- Finite Element Analysis: a classical finite element analysis of the initial design domain is performed.

3- Calculate Strain Energy Density (SED): the strain energy density of the elements and nodes are calculated.

4- Calculation of the Redistribution Factor (RF) and Minimum SED Level (MSL): the redistribution factor is calculated by increasing the value from the last iteration and MSL increases by increasing RF.

5- Extraction of the boundary of the design: the boundary of the design is obtained from the intersection of the SED distribution and MSL.

6- Convergence check: the convergence of the optimization algorithm (such as target volume) is checked by comparing the convergence criteria with the defined convergence threshold. If the convergence condition is satisfied, the algorithm jumps to step 9, otherwise it progresses to step 7.

7- X-FEM structural analysis: an X-FEM analysis is performed on the fixed grid design domain. Using nodal SED numbers, the elements are categorized into three groups: solid, void and boundary elements. Solid and void elements are treated using classical finite element approximation. The stiffness matrix of the boundary elements are calculated by partitioning the solid sub-domain into several sub-triangles and applying gauss quadrature integration scheme described in section 3.2. The global stiffness matrix is calculated by assembling the element stiffness matrices.

8- $\quad$ Go to step 3.

9- Stop the optimization process. 


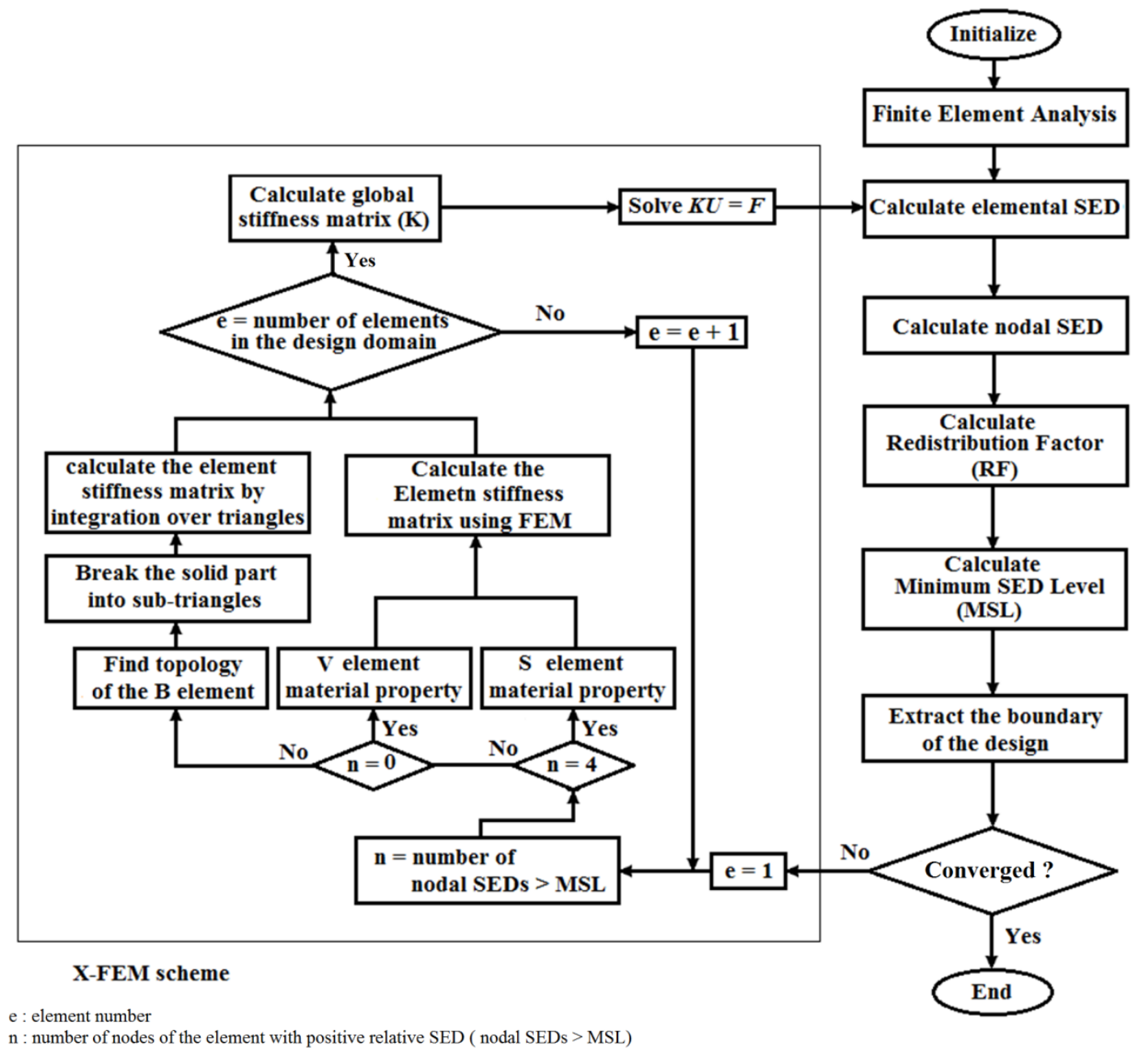

Figure 9. Flowchart of optimization algorithm.

\section{Numerical Examples}

The proposed method of combining X-FEM and evolutionary optimization algorithm was implemented in a MATLAB code to present the topology optimization of 2D rectangular domains as a first validation stage prior to full 3D implementation. Two test cases are used in this study (figure 10). A consistent dimensionless set of parameters are used for both test cases. Test case 1 was a short cantilever beam having length 60, height 30 and thickness 1 where a unit concentrated load is applied in the middle of the free end (symmetric case). Test case 2 was a cantilever beam having the same dimensions as test case 1 but with the load applied at the bottom of the free end (non-symmetric case). The material properties of the solid material were Young's modulus $E=1$ and Poisson's ratio $v=0.3$. The target volume was $50 \%$ of the initial design domain. To avoid singularity issues with the concentrated loading, the strain energy inside the loading regions shown in figure 8 were not used in 
the calculation of the total strain energy and the tip displacements were measured from outside the loading region along the line of loading.
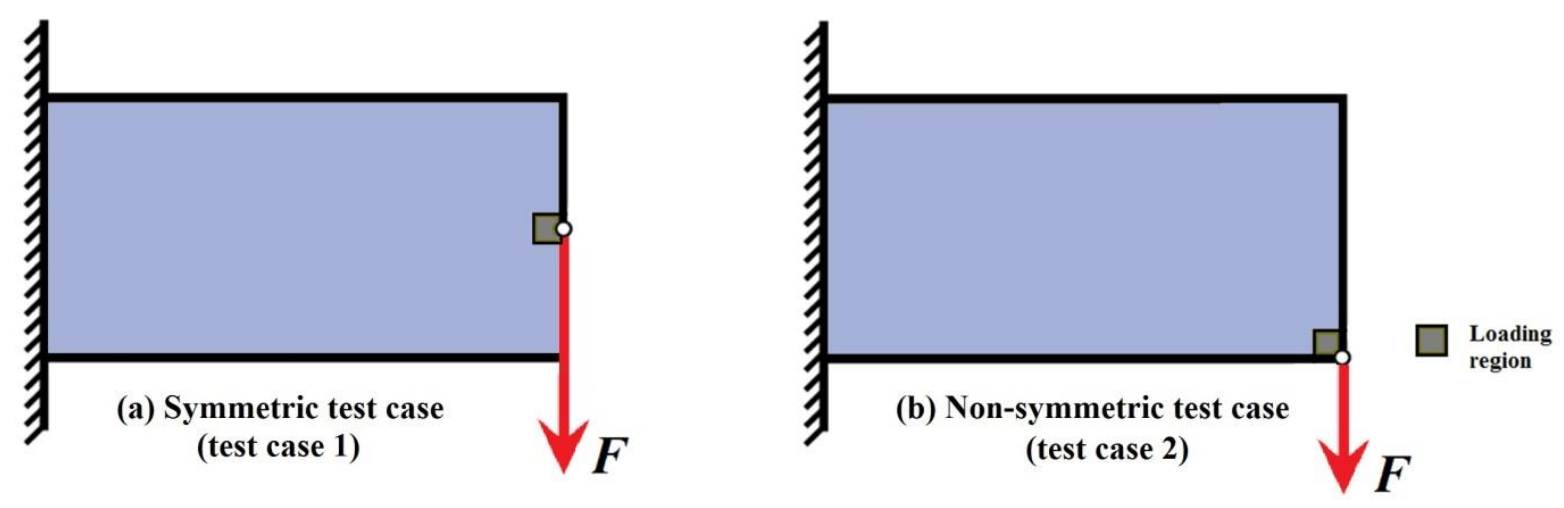

Figure 10. The two test cases.

\subsection{Preliminary examination of convergence}

The initial design domain was descritized using a 60x30 mesh. The optimization started with a fully solid design domain. The evolution histories of the objective function and volume fraction for test cases 1 and 2 are shown in figure 11. It can be seen that the strain energy increases, as material is gradually removed from the design domain, then reaches a constant value at convergence.

The development of the topology in the iterative optimization processes for the two test cases are illustrated in figures 12 and 13. It can be seen that initially a number of holes appear as the volume fraction decreases. After a certain number of iterations some of the holes merge to make larger holes, thus reducing the final complexity of the topology. It can be seen in figure 12 that the topology remains symmetric throughout the optimization. It can be seen in the final topologies that despite using a coarse mesh for this optimization problem, the final designs have clearly defined, smooth boundaries and need no further interpretation (unlike standard SIMP and ESO/BESO methods). In the next two sections, the accuracy of the results are studied and the method is compared with standard BESO. 


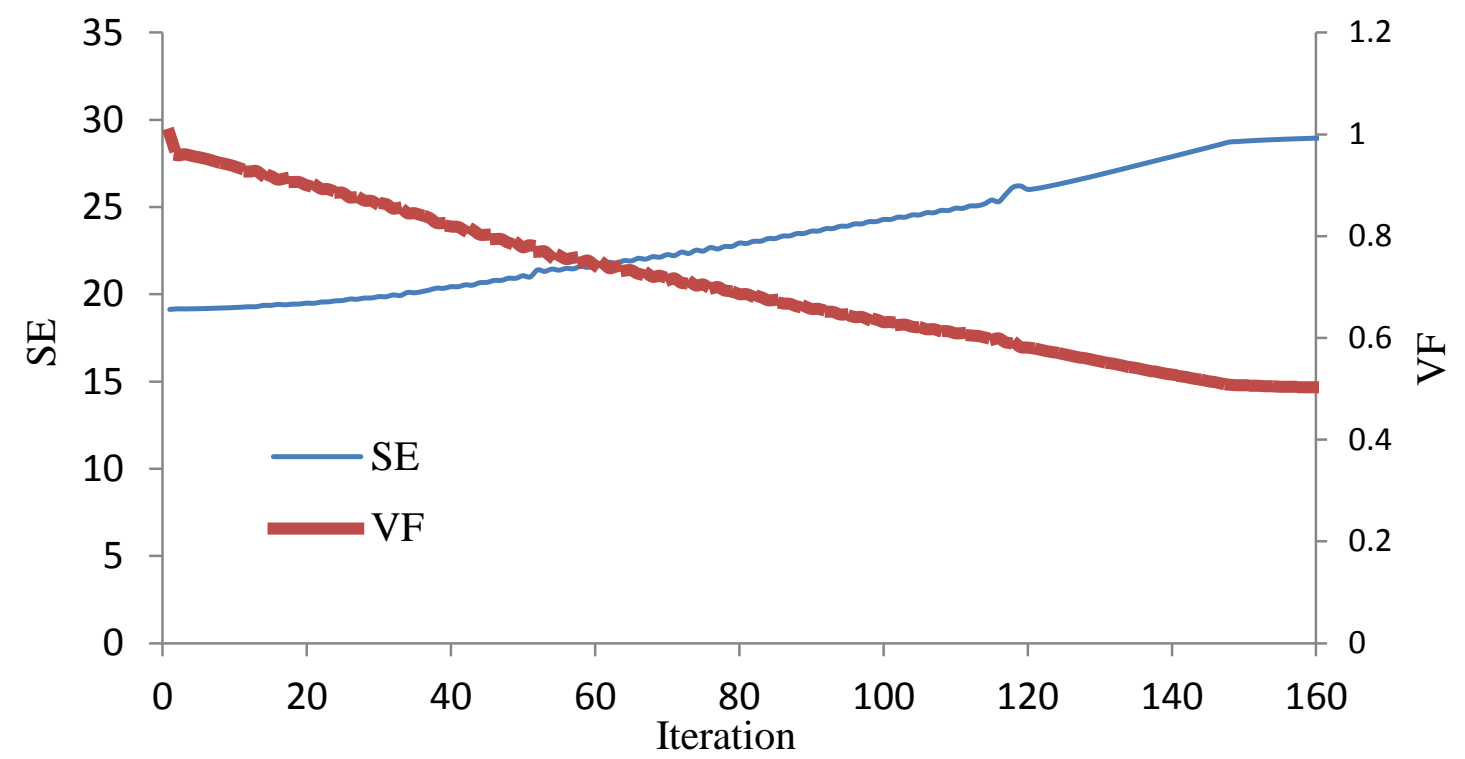

(a)

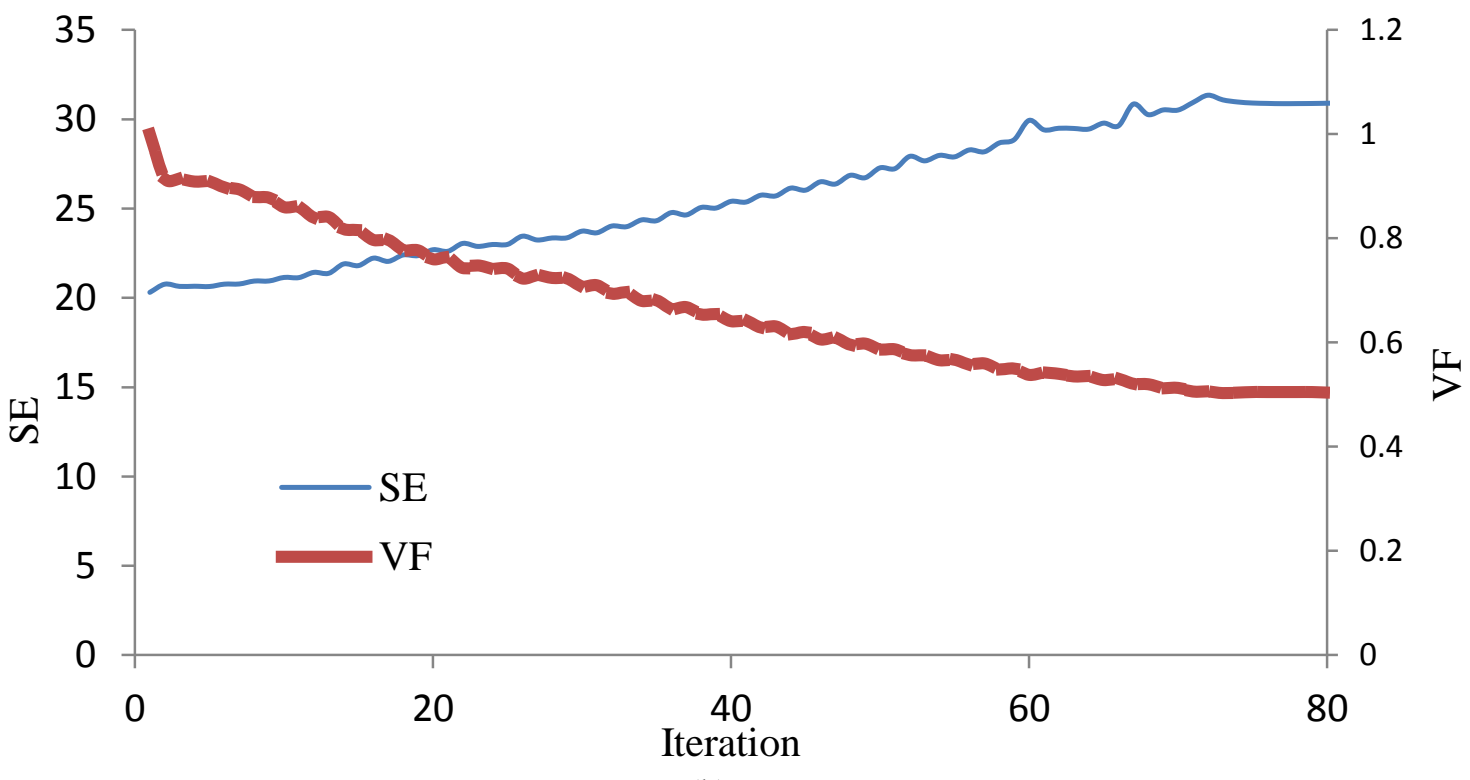

(b)

Figure 11. Evolution history of objective function, SE, and volume fraction (VF) for (a) Test case 1 and (b) Test case 2 . 


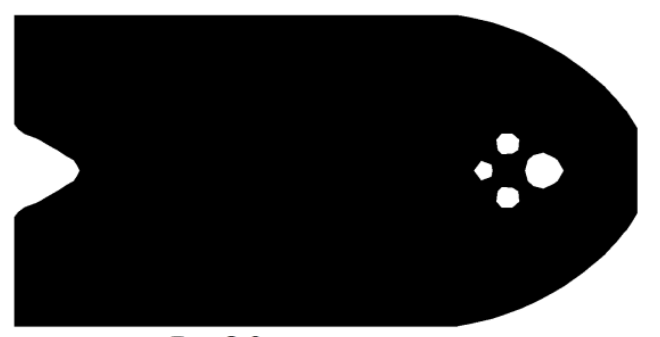

It. 20

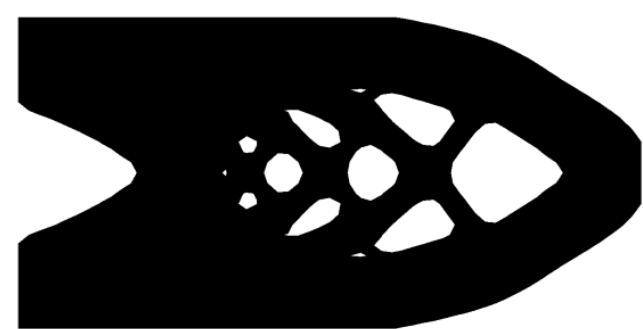

It. 60

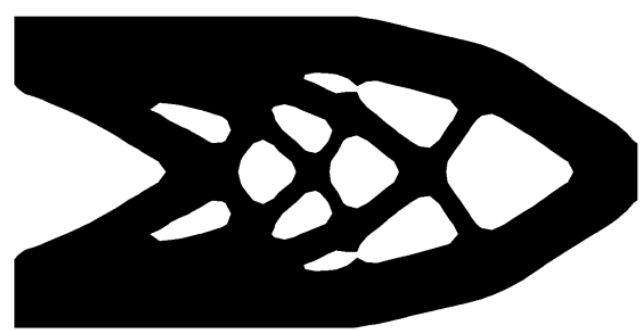

It. 100

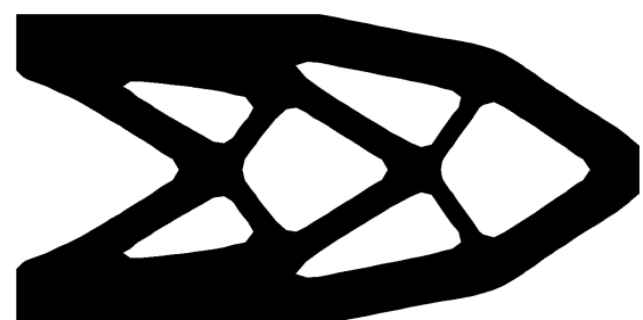

It. 140

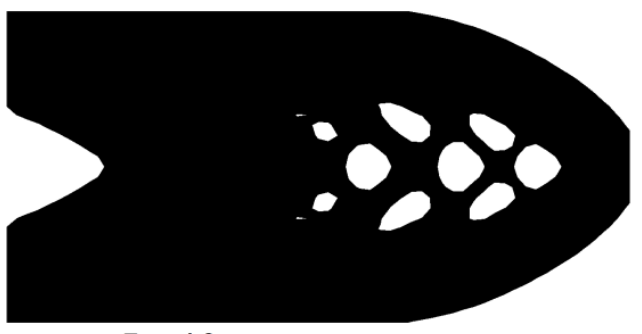

It. 40

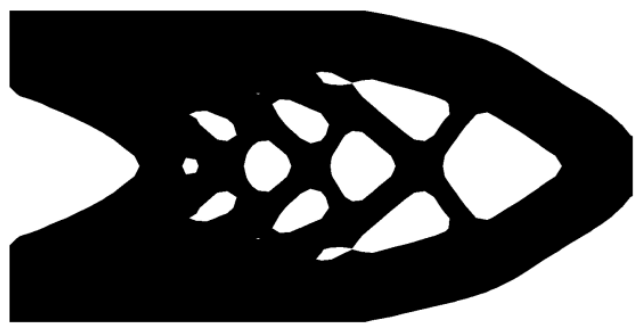

It. 80

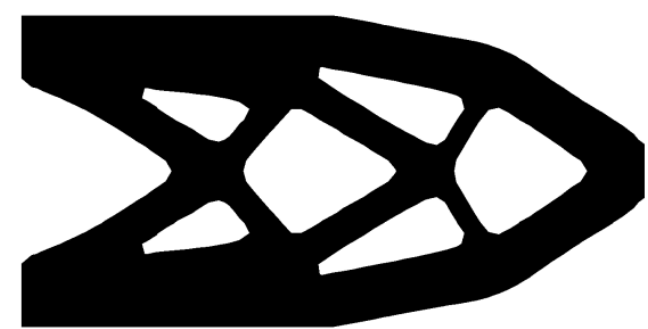

It. 120

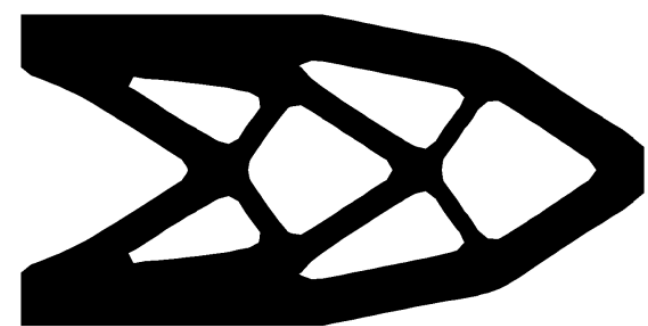

It. 160 (Optimized Design)

Figure 12. Evolution of topology in test case 1. 


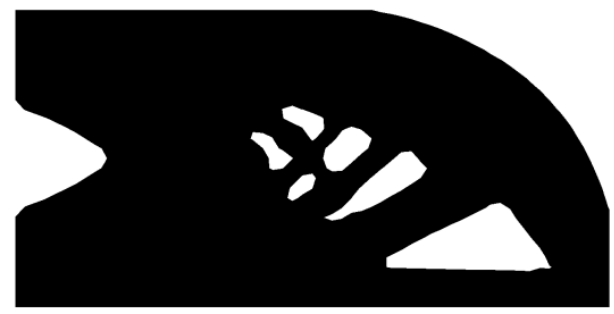

It. 10

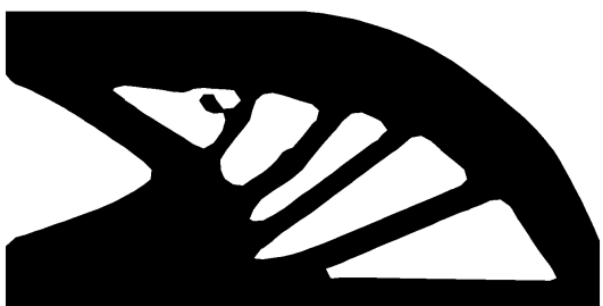

It. 30

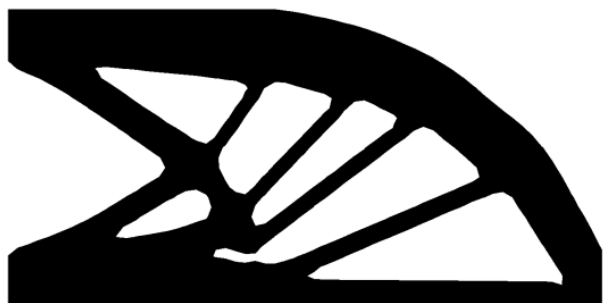

It. 50

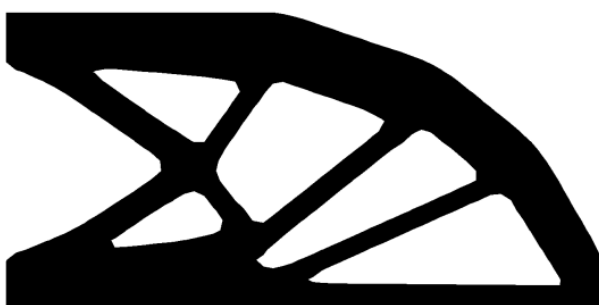

It. 70

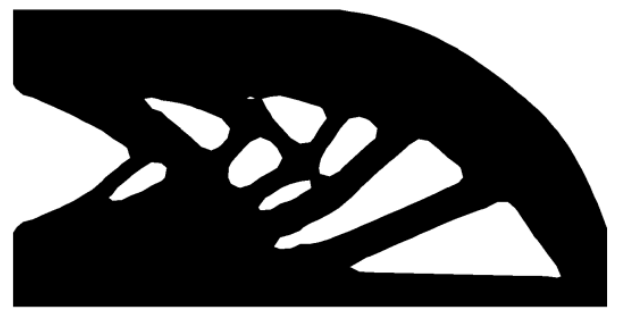

It. 20

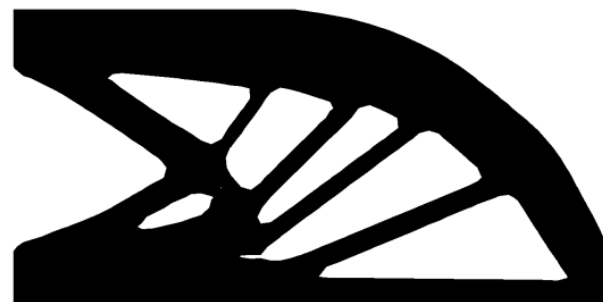

It. 40

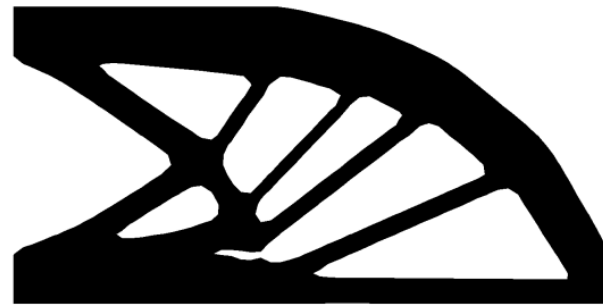

It. 60

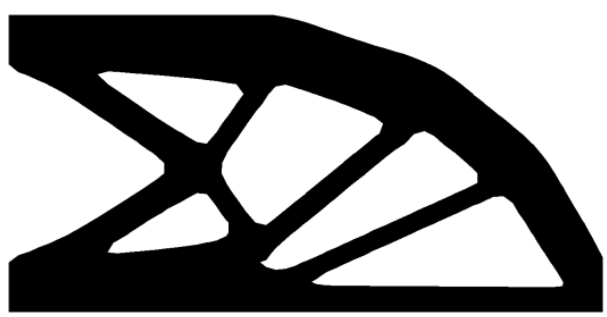

It. 80 (Optimized Design)

Figure 13. Evolution of topology in test case 2.

\subsection{Evaluating X-FEM solutions}

In order to accurately evaluate the performance of the final solutions and the accuracy of the proposed method, the obtained solutions were discretized by a converged, fine structured finite element mesh and solved using the commercial finite element solver NASTRAN from MSC Software (Santa Ana, California, USA) (figure 14).

Table 1 compares the X-FEM solutions and the converged NASTRAN solutions in terms of their strain energies and tip displacements. It can be seen that the X-FEM solutions are very close to the regenerated NASTRAN 
solutions with percentage error less than 0.7 . The small difference in the X-FEM and NASTRAN results may be attributed to the different mesh size used in the two approaches and would be expected to decrease by reducing the mesh size of the design space. However it could be argued that the accuracy obtained using the coarse mesh is sufficient for the topology optimization and the added accuracy of a finer mesh will unnecessarily increase computational time. This is an increasingly important consideration when the method is used for the optimization of real-life 3D structures (as will be shown in future works).

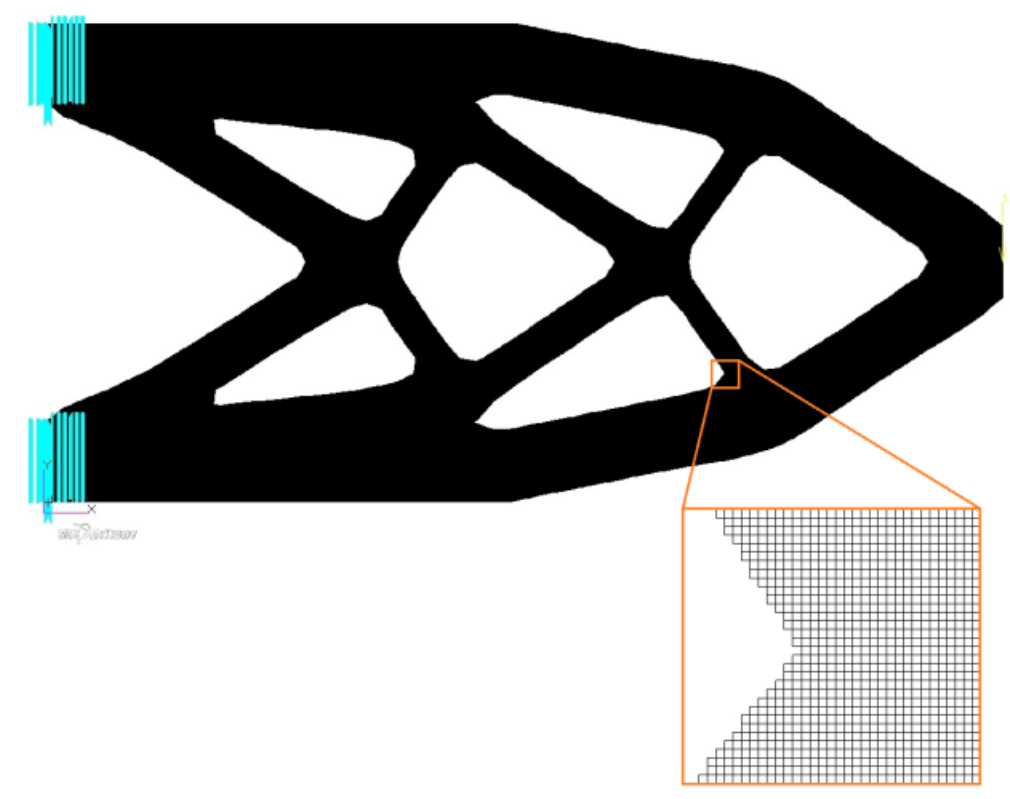

Figure 14. XFEM solution discretized using a converged, fine mesh and imported to NASTRAN for solution.

Table 1. Comparison of X-FEM solutions and regenerated NASTRAN structures.

\begin{tabular}{|l|c|c|}
\hline Test case 1 & Strain Energy & Tip Displacement \\
\hline X-FEM & 29.81 & 57.08 \\
\hline NASTRAN & 30.01 & 57.36 \\
\hline \% Error & 0.67 & 0.49 \\
\hline Test case 2 & Strain Energy & Tip Displacement \\
\hline X-FEM & 30.82 & 61.77 \\
\hline NASTRAN & 31.04 & 62.10 \\
\hline \% Error & 0.70 & 0.53 \\
\hline
\end{tabular}

\subsection{Comparison with BESO solutions}

The solutions obtained from the proposed method are compared with BESO solutions for a range of mesh sizes The BESO solutions were obtained using a soft-kill BESO MATLAB code (Huang and Xie, 2009). In order to overcome the checkerboard problem (Jog and Harber, 1996) in the BESO solutions and retain the complexity of 
the converged solutions, a small filter radius of 1.2 times the element length, was used. The selected evolution rate for BESO was 0.004 which was chosen to give approximately the same number of iterations to converge as the XFEM-Isoline optimization approach (180-200 iterations).

\subsubsection{Comparison of strain energies}

Comparing the topologies obtained using the two approaches for test case 1 (the symmetric problem) one may notice that the converged solutions for the same mesh size have similar topologies (table 2); however the BESO solutions tend to have higher strain energies than the X-FEM solutions. The probable reason for this is the poorer edge representation in BESO method, which has reduced the performance of the converged solutions. At high mesh density the two methods had very similar performance, as would be expected, however, this would obviously be more computationally expensive. To increase the performance of these BESO solutions, additional post-processing is required to smooth the boundaries. In test case 2, which is a non-symmetric problem, the two approaches have generated different topologies (table 3). The strain energies of the X-FEM solutions are again lower than the BESO ones, indicating better performance for the X-FEM solutions.

It can be seen in tables 2 and 3 that both methods result in final topologies that are mesh dependent, which is generally the case for element based topology optimization methods. Pseudo mesh-independent topologies can be obtained by the use of coarsening actions such as filtering the sensitivities or increasing the filter radius (Huang and Xie, 2007). However these methods still have their limits in terms of mesh independency and will tend to result in coarser solution that can have lower performance than more refined solutions. Generally speaking, increasing the mesh density increases the complexity of the converged solutions and reduces the strain energies. Therefore increasing the mesh density can improve the performance of the final optimized result. This issue has also been studied in earlier investigations for BESO (Aremu et al, 2012). Figure 15 illustrates the strain energy of the converged solution as a function of mesh density for test case 2. It shows that X-FEM optimization approach is more robust than the BESO method and that the strain energies of the X-FEM solutions converged earlier than BESO ones. However, as the mesh gets finer the strain energies of BESO solutions get closer to the X-FEM solutions. It can be seen that increasing the mesh density results in the strain energies produced using XFEM-Isoline approach and NASTRAN FEA become close, relative to the fluctuations in the data beyond $1 / \mathrm{h}=80$. 
Table 2. X-FEM and BESO solutions of test case 1 for a range of mesh sizes.

\begin{tabular}{|c|c|c|c|c|c|}
\hline Mesh & $40 \times 20$ & $60 \times 30$ & $80 \times 40$ & $100 \times 50$ & $120 \times 60$ \\
\hline X-FEM & & & & & \\
\hline $\mathrm{SE}$ & 29.49 & 29.04 & 28.91 & 28.85 & 28.88 \\
\hline BESO & & & & & \\
\hline SE & 31.55 & 30.34 & 30.08 & 30.06 & 29.80 \\
\hline
\end{tabular}

Table 3. X-FEM and BESO solutions of test case 2 for a range of mesh sizes.

\begin{tabular}{|c|c|c|c|c|c|}
\hline Mesh & $40 \times 20$ & $60 \times 30$ & $80 \times 40$ & $100 \times 50$ & $120 \times 60$ \\
\hline X-FEM & & & & & \\
\hline SE & 31.08 & 30.82 & 30.74 & 30.54 & 30.64 \\
\hline BESO & & & & & \\
\hline SE & 32.91 & 32.05 & 31.54 & 31.72 & 31.57 \\
\hline
\end{tabular}




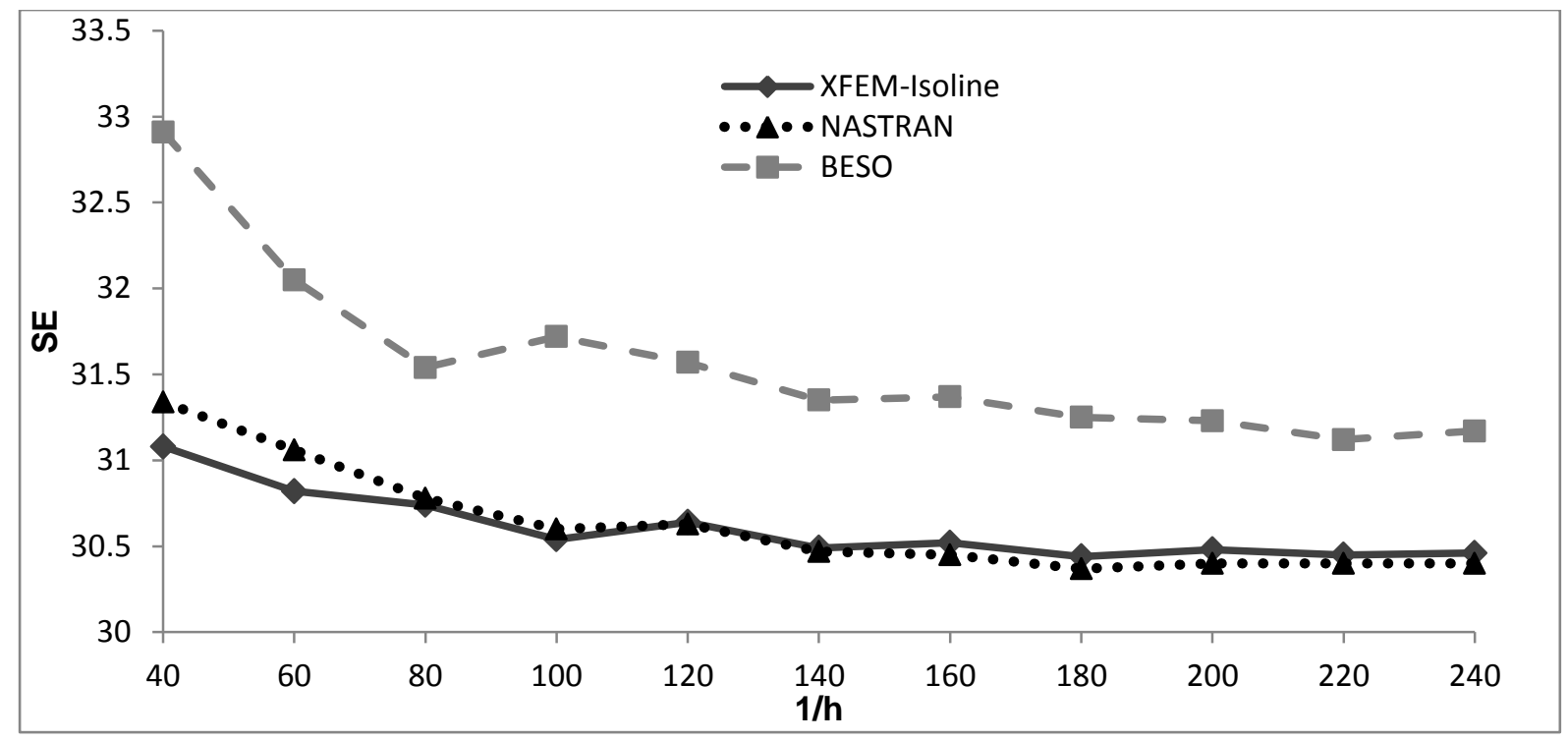

Figure 15. Changes in strain energy (SE) by reducing the mesh size (h) in test case 2.

Table 4 compares the computational time for the two optimization methods for test case 1 after 100 iterations. It can be seen that at low mesh densities BESO is much faster than XFEM, however, the computational time ratio decreases by increasing the mesh density. It is shown in the paper that BESO solutions require higher mesh densities and more post-processing to obtain a smooth topology, therefore the total time for design could be decreased by using the XFEM-Isoline method.

Table 4. Comparison of the time cost of BESO and X-FEM for 100 iterations

\begin{tabular}{|l|c|c|c|c|c|}
\hline Approach $\backslash$ Mesh & $40 \times 20$ & $60 \times 30$ & $80 \times 40$ & $100 \times 50$ & $120 \times 60$ \\
\hline X-FEM & $19 \mathrm{~s}$ & $43 \mathrm{~s}$ & $92 \mathrm{~s}$ & $188 \mathrm{~s}$ & $434 \mathrm{~s}$ \\
\hline BESO & $9 \mathrm{~s}$ & $25 \mathrm{~s}$ & $65 \mathrm{~s}$ & $151 \mathrm{~s}$ & $399 \mathrm{~s}$ \\
\hline Ratio & $211 \%$ & $172 \%$ & $142 \%$ & $125 \%$ & $109 \%$ \\
\hline
\end{tabular}

\subsubsection{Comparison of surface roughness}

As a post processing stage, a Laplacian smoothing algorithm can be used to create smoother boundaries for the optimised topologies, if this is required for manufacture for instance. Laplacian smoothing is an iterative smoothing technique, commonly used in image processing and improving the quality of finite element meshes (Cannan et al, 1993; Freitag, 1997). In the image processing application, Laplacian smoothing operates by replacing the grey value of a pixel with an average of the grey values of neighbouring pixels. In the FE meshing application, the location of a vertex is modified using the average of the locations of neighbouring vertices (Vollmer et al, 2001). Figure 16 shows the boundaries before and after smoothing for the BESO solutions of test case 2 for a range of mesh densities. The average surface roughness of the optimized topologies before smoothing and the number of iterations in the Laplacian smoothing are also included in the figure. The root 
mean square roughness $\left(\mathrm{R}_{\mathrm{q}}\right)$ was determined by comparison of the topology boundaries before and after the Laplacian smoothing. It can be seen in Figure 16 that the surface roughness of the topologies from the BESO optimization increases as the mesh density decreases. Figure 17 shows the boundaries of X-FEM solutions for the same test case before and after smoothing. Compared to the BESO solutions, the surface roughness of the XFEM solutions are much lower and they need far fewer iteration steps in the Laplacian smoothing. Also it can be seen that unlike the BESO solutions, the surface roughness of the X-FEM solutions have little dependency on the mesh density. 


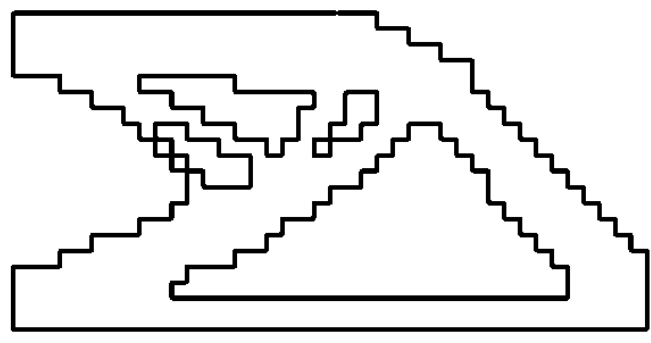

mesh $40 \times 20 \quad R_{q}=0.363$

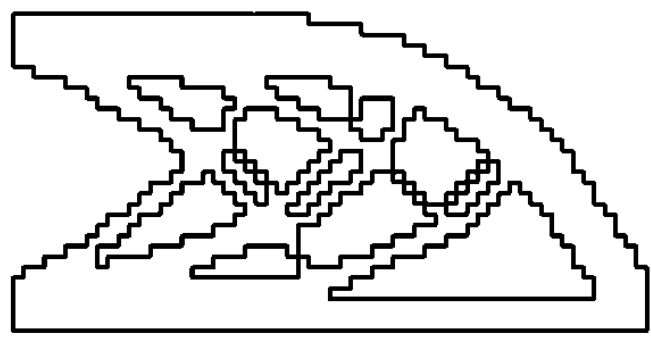

mesh $60 \times 30 \quad R_{q}=0.270$

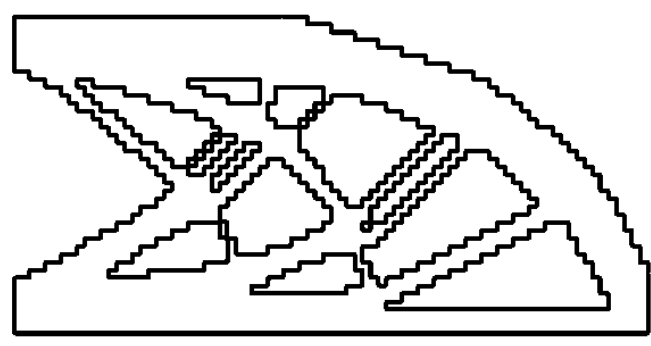

$\begin{array}{ll}\text { mesh } 80 \times 40 & R_{\mathrm{q}}=0.198\end{array}$

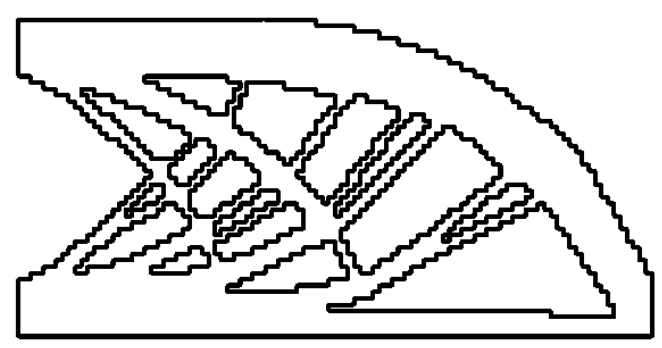

mesh $100 \times 50 \quad R_{q}=0.165$

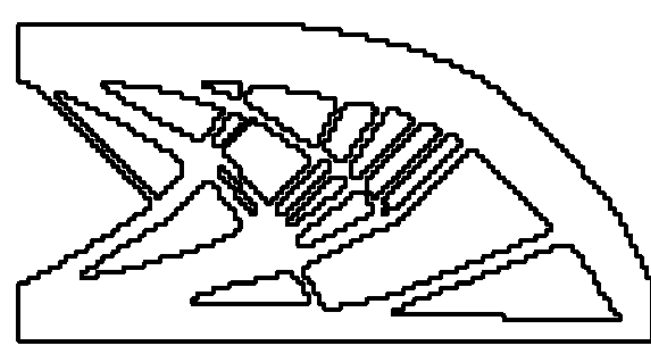

mesh $120 \times 60$

$R_{q}=0.136$

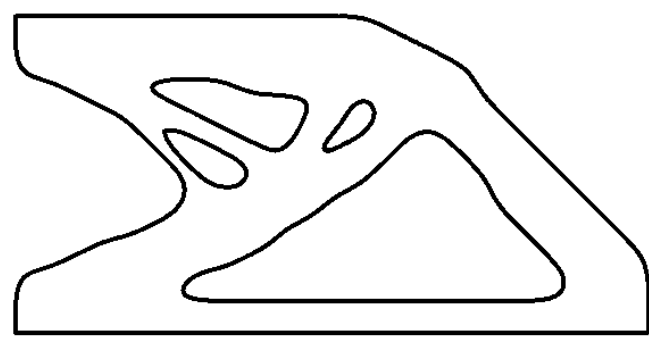

It. 1500

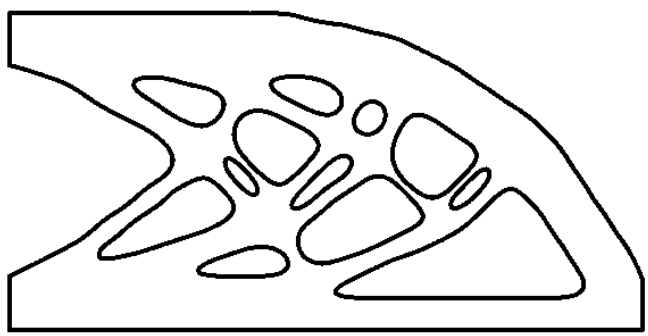

It. $\mathbf{1 1 0 0}$

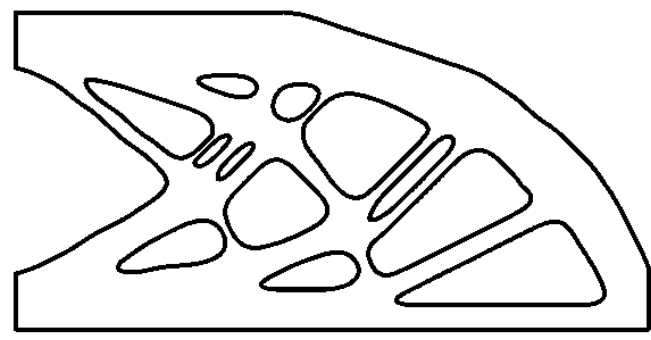

It. 800

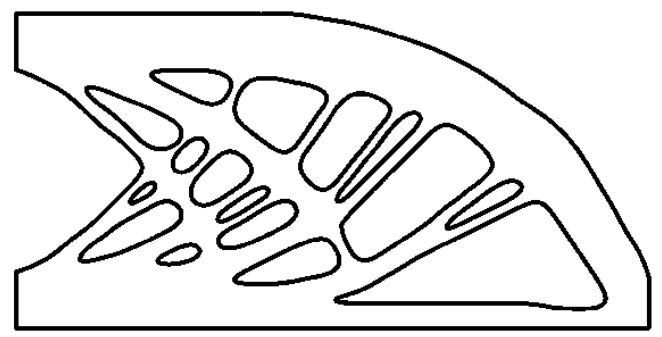

It. 600

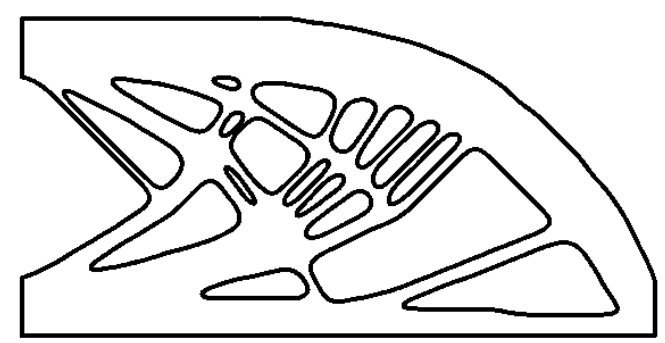

It. $\mathbf{5 0 0}$

Figure 16. BESO solutions before/after smoothing. 
Before Smoothing

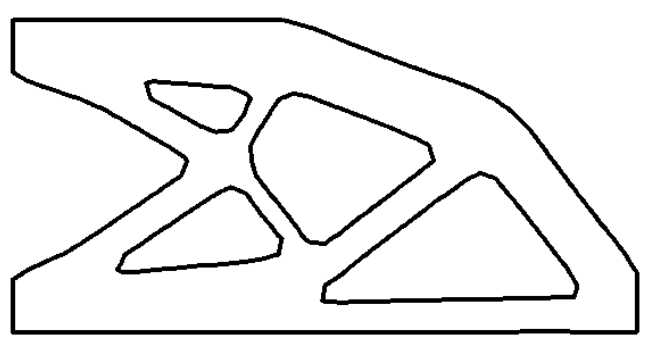

mesh $40 \times 20$

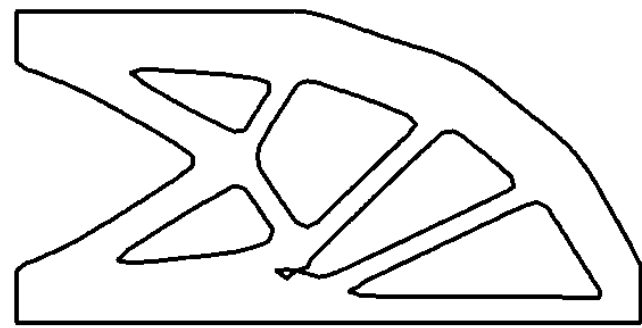

$\begin{array}{ll}\text { mesh } 60 \times 30 & R_{\mathrm{q}}=0.0157\end{array}$

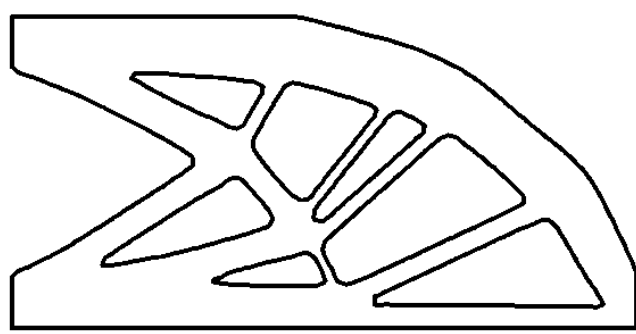

mesh $80 \times 40 \quad R_{q}=0.0165$

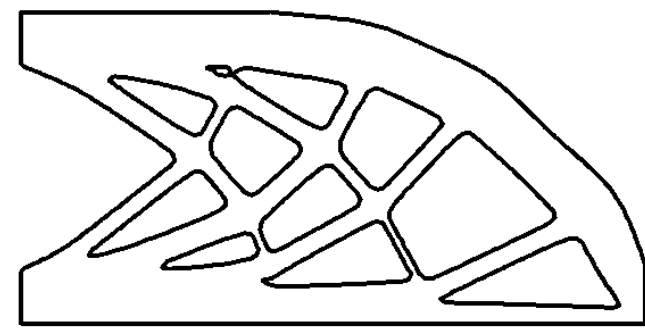

mesh $100 \times 50 \quad R_{q}=0.0166$

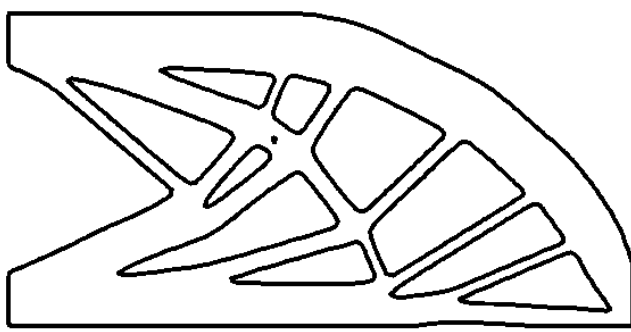

mesh $120 \times 60$

$R_{q}=0.0165$
After Smoothing

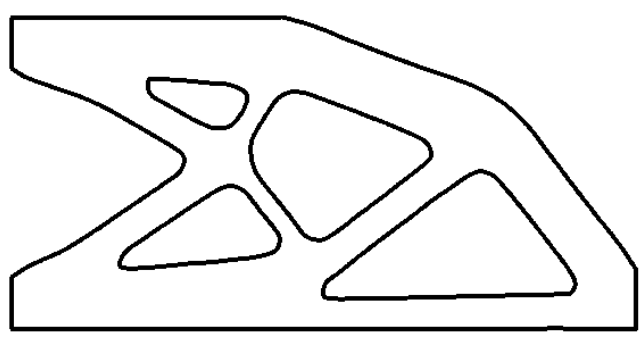

It. 50

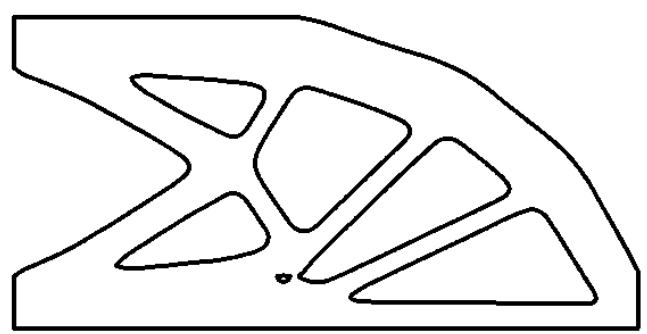

It. 50

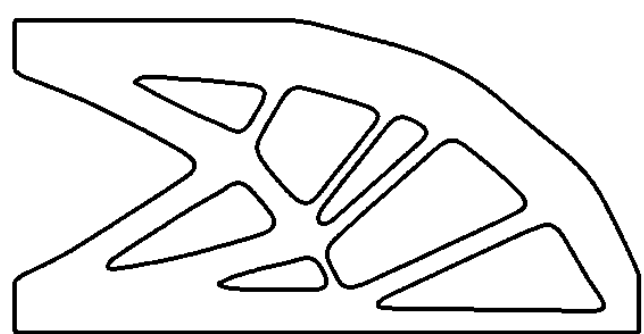

It. 50

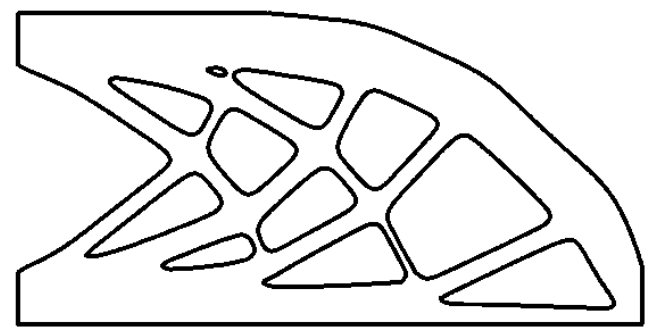

It. $\mathbf{5 0}$

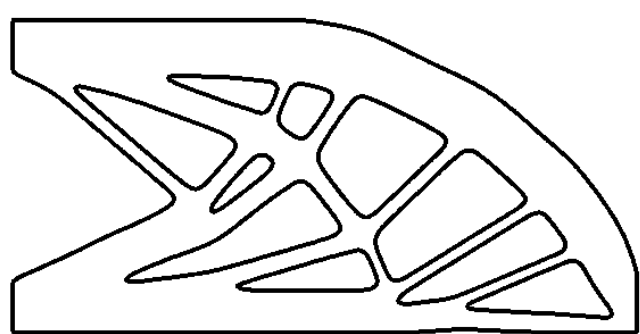

It. 50

Figure 17. X-FEM solutions before/after smoothing. 


\section{Conclusions}

In this study, the X-FEM and Isoline design methods are combined with an evolutionary optimization algorithm to determine the topology optimization of $2 \mathrm{D}$ continuum structures. Our results suggest that using X-FEM has significant advantages, not only does it avoids time consuming remeshing techniques, but also generates structures that have smooth boundaries requiring little or no further interpretation or post processing. Using simple test geometries, it has been shown that X-FEM based topology optimization has the potential for greater accuracy and more robust solutions with less dependence on mesh size than BESO, though this needs to be established for more realistic, 3D geometries. It is anticipated however that this method is relatively simple to extend. A possible route to achieving this may involve using 8-node brick elements where the solid part of the boundary element could be represented by sub-tetrahedrons.

\section{Acknowledgements}

The authors are grateful for the funding provided by the University of Nottingham and Loughborough University.

\section{References}

Allaire, G., Jouve, F., Toader, A. M., 2004. Structural optimisation using sensitivity analysis and a level set method, J. Comp. Phys., 194, pp. 363-393.

Aremu, A., Ashcroft, I., Wildman, R, Hague, R., Tuck, C., Brackett, D., 2012. The effects of BESO parameters on an industrial designed component for additive manufacture. Proc IMechE Part B: J Engineering Manufacture, in press.

Bendsøe, M.P. 1989. Optimal shape design as a material distribution problem. Struct. Optim. 1, pp. 193-202.

Bendsøe, M.P. and Kikuchi, N., 1988. Generating optimal topologies in structural design using a homogenization method. Computer Methods in Applied Mechanics and Engineering, 71, pp. 197-224.

Belytschko, T. and Black, T, 1999. Elastic crack growth in finite elements with minimal remeshing. International Journal for Numerical Methods in Engineering, 45 (5), pp 601-620.

Canann, S., Stephenson, M. and Blacker, T., 1993. Optismoothing: An optimization-driven approach to mesh Smoothing. Finite Elements in Analysis and Design, 13, pp. 185-190.

Chu, D.N., Xie, Y.M., Hira, A. and Steven, G.P.,1996. Evolutionary structural optimisation for problems with stiffness constraints. Finite Elements in Analysis and Design, 21, pp. 239-51.

Dunning, P., Kim, H. A. and Mullineux, G., 2008. Error analysis of fixed grid formulation for boundary based structural optimisation. In: 7th ASMO UK / ISSMO conference on Engineering Design Optimisation, 7-8 July 2008, Bath, UK.

Freitag, L., 1997. On combining Laplacian and optimization based mesh smoothing techniques. Proceedings of the 1997 Joint Summer Meeting of American Society of Mechanical Engineers (ASME) American Society of Civil Engineers (ASCE) and Society of Engineers Science (SES), pp. 37-44.

Gerstenberger, A., Wall. W. A., 2008. An eXtended finite element method/Lagrange multiplier based approach for fluidstructure interaction. Computer Methods in Applied Mechanics and Engineering, 197, pp.1699-714.

Huang, X. and Xie, Y.M., 2007. Convergent and mesh-independent solutions for bi-directional evolutionary structural optimisation method. Finite Elements in Analysis and Design, 43(14), pp. 1039-49.

Huang, X., Xie, Y. M., 2009. Evolutionary Topology Optimisation of Continuum Structures, Wiley. 
Jog, C.S. and Harber, R.B., 1996. Stability of finite element models for distributed-parameter optimization and topology design. Comput. Meth. Appl. Mech. Engng. 130: 1951-65.

Lee, D., Park, S., Shin, S., 2007. Node-wise topological shape optimum design for structural reinforced modeling of Michell-type concrete deep beams. J Solid Mech Mater Eng, 1(9), pp. 1085-96.

Maute, K., Ramm, E., 1995. Adaptive topology optimisation. Struct Optim, 10, pp. 100-12.

Melenk, J.M. and Babuška, I., 1996. The partition of unity finite element method: Basic theory and applications. Computer Methods in Applied Mechanics and Engineering, 139, pp. 289-314.

Miegroet, L.V., Duysinx, P., 2007. Stress concentration minimization of 2D Filets using X-FEM and level set description. Structural and Multidisciplinary Optimisation, 33, pp. 425-38.

Moës, N., Dolbow, J. and Belytschko, T., 1999. A finite element method for crack growth without remeshing, International Journal for Numerical Methods in Engineering. 46, pp. 131-150.

Querin, O.M., Steven, G.P. and Xie, Y.M., 1988. Evolutionary structural optimisation (ESO) using a bidirectional algorithm. Engineering Computations, 15 ( 8), pp. 1031-1048.

Sigmund, O., 2001. A 99 line topology optimisation code written in Matlab. Struct Multidiscipl Optim, 21, pp. 120-127.

Sukumar, N., Chopp, D. L., Moës, N. and Belytschko, T., 2001. Modeling Holes and Inclusions by Level Sets in the Extended Finite Element Method. Computer Methods in Applied Mechanics and Engineering, 190, pp. 6183-6200.

Victoria, M., Martı', P., Querin, O.M., 2009. Topology design of two-dimensional continuum structures using isolines. Computer and Structures, 87 , pp.101-109.

Victoria, M., Querin, O.M., Mart1', P., 2010. Topology design for multiple loading conditions of continuum structures using isolines and isosurfaces. Finite Elements in Analysis and Design, 46 , pp. 229-237.

Vollmer, J., Mencl, R. and Müller, H., 2001. Improved Laplacian Smoothing of Noisy Surface Meshes. Computer Graphic Foroum, 18(3), pp. 131-138.

Wang, M.Y., Wang, X., Guo, D., 2003. A level set method for structural topology optimisation. Comput. Meth .Appl. Eng., 192, pp.227-46.

Wei, P., Wang, M.Y., Xing, X., 2010. A study on X-FEM in continuum structural optimization using level set method. Computer-Aided Design, 42, pp. 708-719.

Xie, Y. M. and Steven, G.P., 1993. A simple evolutionary procedure for structural optimization. Computers \& Structures, 49, pp. $885-896$

Yang, X. Y., Xie, Y. M., Steven, G. P., and Querin, O. M., 1999. Bidirectional evolutionary method for stiffness optimisation. AIAA J., 37(11), pp.1483-1488.

Zhou, M., Rozvany, G.I.N., 1991. The COG algorithm, Part II: Topological, geometrical and general shape optimisation. Comp. Meth. Appl. Mech. Eng., 89, pp. 309-336. 